\title{
Synthesis of 9-Substituted Derivatives of tert-Butyl 6-(9h-Purin-6-Ylthio) Hexylcarbamate
}

\author{
Venkateswara Rao $\mathbf{P}^{1 *}$, Ravindhranath $\mathrm{K}^{2}$ and Ravi Kumar $\mathrm{K}^{3}$ \\ ${ }^{1}$ R. V.R and J.C. College of Engineering, Guntur, AP, India \\ ${ }^{2}$ Dept of Engg. Chemistry and P G Chemistry, Bapatla Engineering College, Baptla, AP, India \\ ${ }^{3} R A$ Chem Pharma Limited, R\&D Division, Prasanth Nagar, Hyderabad, AP, India
}

\begin{abstract}
We report herein the synthesis of series of 9-substituted derivatives of tert-butyl 6-(9H-purin-6-ylthio) hexylcarbamate by the reaction of tert-butyl 6-(9H-purin-6-ylthio) hexylcarbamate with different acid chlorides using triethylamine in Dichloromethane solvent.
\end{abstract}

Keywords: 6-mercaptopurine; Tert-butyl 6-(9H-purin-6-ylthio) hexylcarbamate; Acylation; Dichloromethane; Boc-deprotection

\section{Introduction}

The purine ring system possesses undisputed biological importance and it is considered to be one of the most important heterocyclic rings in nature [1]. Cells obtain purine nucleotides through two separate metabolic pathways, de novo purine synthesis and salvage of extracellular purine bases and nucleotides [2]. Therefore, de novo purine synthesis and several enzymes involved in the purine metabolism pathway are important targets for antimetabolites [2]. Purine antimetabolites have been used in the development of many potent medicinal agents, which exhibited antineoplastic, antileukemic, antiviral, antibacterial, and antifungal activities [1-4]. The purine nucleoside analogs are also used in the treatment of autoimmune diseases [5]. 6-mercapto purine is used therapeutically as an immunosuppressive agent [6] and inhibits the growth of bacterial and mammalian cells [7]. Other 6-mercapto purine, mercapto pyridine and mercapto pyrimidine derivatives also exhibit antibacterial activity, and have been studied as agents for targeting melanoma [8], reducing cholesterol and as vasodilators [9]. As part of our present research work we are preparing acylated derivatives of tertbutyl 6-(9H-purin-6-ylthio) hexylcarbamate. According the Neiman and Bergmann [10] work we confirmed that acylation at the 9-position.

\section{Materials and Methods}

All ${ }^{1} \mathrm{H}$ NMR spectra were recorded on $400 \mathrm{MHz}$ Varian FT-NMR spectrometers. All chemical shifts are given as $\delta$ value with reference to Tetra methyl silane (TMS) as an internal standard. Melting points were taken in open capillaries. The IR spectra were recorded on a PerkinElmer 257 spectrometer using $\mathrm{KBr}$ discs. Products were purified by flash chromatography on 100-200 mesh silica gel. The chemicals and solvents were purchased from commercial suppliers either from Aldrich, Spectrochem and they were used without purification prior to use.

\section{Scheme of synthesis}

The synthesis of the title compounds $(8 \mathrm{a}-8 \mathrm{i})$ were prepared by the acylation of compound 6 by using different acid chlorides. The main intermediate compound 6 was prepared from 6- mercaptopurine.

\section{Experimental}

\section{Synthesis of tert-butyl 6-hydroxyhexylcarbamate (2)}

To a stirred solution of compound $1(5 \mathrm{gm}, 42.73 \mathrm{mmol})$ in THF $(50 \mathrm{ml})$ was added (Boc) $\mathrm{O}(9.8 \mathrm{~mL}, 42.73 \mathrm{mmol})$ at $0^{\circ} \mathrm{C}$ for $30 \mathrm{~min}$ and at $\mathrm{rt}$ for over $1 \mathrm{~h}$. The reaction mixture was evaporated in vacuum until dryness. The obtained crude reaction product $2(8 \mathrm{gm})$ was used to next step without any further purification.

\section{Synthesis of 6-(tert-butoxycarbonylamino) hexyl methane-} sulfonate (3)

To a stirred solution of compound $2(8 \mathrm{gm}, 36.86 \mathrm{mmol})$ in THF $(50 \mathrm{ml})$ was added DIPEA $(20.3 \mathrm{~mL}, 110.5 \mathrm{mmol})$, methane sufonyl chloride $(4.5 \mathrm{~mL}, 55.29 \mathrm{mmol})$ at $0^{\circ} \mathrm{C}$ for $30 \mathrm{~min}$ and at $\mathrm{rt}$ for over $2 \mathrm{~h}$. Reaction was monitored by TLC. When reaction was completed the mixture was diluted with ethyl acetate and washed with $\mathrm{H}_{2} \mathrm{O}$, brine solution. Organic layer was dried over $\mathrm{Na}_{2} \mathrm{SO}_{4}$, concentrated under reduced pressure. Crude compound was purified by column chromatography to obtained compound $3(9 \mathrm{~g}, 71 \%)$ as a light yellowish gummy solid. ${ }^{1} \mathrm{H}$ NMR (DMSO-d $): \delta 6.76(\mathrm{~m}, 1 \mathrm{H}), 4.17(\mathrm{t}, \mathrm{J}=8.4 \mathrm{~Hz}$ $2 \mathrm{H}), 3.15(\mathrm{~s}, 3 \mathrm{H}), 2.89(\mathrm{q}, \mathrm{J}=8.4 \mathrm{~Hz}, 2 \mathrm{H}), 1.66-1.59(\mathrm{~m}, 2 \mathrm{H}), 1.36(\mathrm{~s}$ 9H), 1.33-1.25 (m, 6H); (LC-MS) m/z: $196.1[\mathrm{M}-100 \text { (Boc) }]^{+}$.

\section{Synthesis of 9H-purine-6-thiol (5)}

To a stirred solution of compound $4(20 \mathrm{gm}, 129.39 \mathrm{mmol})$ in DMF $(200 \mathrm{ml})$ was added potassium thioacetate $(29.5 \mathrm{gm}, 258.79 \mathrm{mmol})$ at room temperature. The reaction mixture was stirred for an additional $6 \mathrm{~h}$ at $80^{\circ} \mathrm{C}$. Reaction was monitored by TLC. When reaction was completed the mixture was cooled to room temperature and diluted with water $(200 \mathrm{~mL})$, ethyl acetate $(200 \mathrm{~mL})$ and stirred for $1 \mathrm{~h}$ at RT. Reaction mixture was filtered and obtained solid was dried under vacuum to get compound $5(16 \mathrm{~g}, 80 \%)$ as a brown colour solid. ${ }^{1} \mathrm{H}$ NMR (DMSO-d $\left.\mathrm{d}_{6}\right): \delta 13.78(\mathrm{~s}, 1 \mathrm{H}), 13.66(\mathrm{~s}, 1 \mathrm{H}), 8.41(\mathrm{~s}, 1 \mathrm{H}), 8.20(\mathrm{~s}$, $1 \mathrm{H})$; (LC-MS) $\mathrm{m} / z: 153.06[\mathrm{M}+\mathrm{H}]^{+}$.

\section{Synthesis of tert-butyl 6-(9H-purin-6-ylthio) hexylcarbamate} (6)

To a stirred solution of compound 5 ( $5 \mathrm{gm}, 32.89 \mathrm{mmol})$ in DMF $(50 \mathrm{ml})$ was added potassium carbonate $(4.5 \mathrm{gm}, 32.89 \mathrm{mmol})$ and compound $3(9.7 \mathrm{gm}, 32.89 \mathrm{mmol})$ at room temperature. The reaction mixture was stirred for an additional $16 \mathrm{~h}$ at RT. Reaction

${ }^{*}$ Corresponding author: Venkateswara Rao P, R.V.R and J.C. College of Engineering, Guntur, AP, India, Tel: +919490642727; E-mail: pvr007.1@gmail.com

Received March 05, 2013; Accepted April 27, 2013; Published April 29, 2013

Citation: Venkateswara Rao P, Ravindhranath K, Ravi Kumar K (2013) Synthesis of 9-Substituted Derivatives of tert-Butyl 6-(9h-Purin-6-Ylthio) Hexylcarbamate. Med chem 3: 188-191. doi:10.4172/2161-0444.1000137

Copyright: (c) 2013 Venkateswara Rao P, et al. This is an open-access article distributed under the terms of the Creative Commons Attribution License, which permits unrestricted use, distribution, and reproduction in any medium, provided the original author and source are credited. 
was monitored by TLC. When reaction was completed the mixture was diluted with ethyl acetate and washed with $\mathrm{H}_{2} \mathrm{O}$, brine solution. Organic layer was dried over $\mathrm{Na}_{2} \mathrm{SO}_{4}$, concentrated under reduced pressure. Crude compound was purified by column chromatography to obtained compound $6(6.5 \mathrm{~g}, 56 \%)$ as an off-white solid, compound $7(600 \mathrm{mg})$ as a light brown solid. Compound 6- ${ }^{1} \mathrm{H}$ NMR (DMSO- $\mathrm{d}_{6}$ ): $\delta 13.50(\mathrm{~s}, 1 \mathrm{H}), 8.71(\mathrm{~s}, 1 \mathrm{H}), 8.42(\mathrm{~s}, 1 \mathrm{H}), 6.78(\mathrm{~s}, 1 \mathrm{H}), 2.95-2.89(\mathrm{~m}$, $2 \mathrm{H}), 1.72-1.68(\mathrm{~m}, 2 \mathrm{H}), 1.38(\mathrm{~s}, 9 \mathrm{H}), 1.36-1.25(\mathrm{~m}, 8 \mathrm{H})$; (LC-MS) $\mathrm{m} / z$ : $352.2[\mathrm{M}+\mathrm{H}]^{+}$. Compound $7-^{-1} \mathrm{H}$ NMR (DMSO-d $): \delta 8.70(\mathrm{~s}, 1 \mathrm{H}), 8.47$ (s, $1 \mathrm{H}), 6.77-6.75(\mathrm{~m}, 2 \mathrm{H}), 4.24-4.21(\mathrm{~m}, 2 \mathrm{H}), 3.60$ (br s, $1 \mathrm{H}), 2.90-2.85$ (m, $4 \mathrm{H}), 1.83-1.68(\mathrm{~m}, 5 \mathrm{H}), 1.36(\mathrm{~s}, 18 \mathrm{H}), 1.36-1.24(\mathrm{~m}, 12 \mathrm{H})$; (LC-MS) $\mathrm{m} / z: 551.4[\mathrm{M}+\mathrm{H}]^{+}$.

\section{General procedure for compound 8a-8i}

To a stirred solution of compound $7(1 \mathrm{mmol})$ in Dichloromethane $(3 \mathrm{ml})$ was added TEA $(3 \mathrm{mmol})$, Acid chloride $(1.2 \mathrm{mmol})$ at $0^{\circ} \mathrm{C}$ for $30 \mathrm{~min}$ and at $\mathrm{rt}$ for over $4 \mathrm{~h}$. Reaction was monitored by TLC. When reaction was completed, the reaction mixture was diluted with DCM and washed with $\mathrm{H}_{2} \mathrm{O}$, brine solution. Organic layer was dried over $\mathrm{Na}_{2} \mathrm{SO}_{4}$, concentrated under reduced pressure. Crude compound was purified by column chromatography to get compound $8 \mathrm{a}-8 \mathrm{i}$.

tert-butyl

6-(9-(2-fluorobenzoyl)-9H-purin-6-ylthio) hexylcarbamate 8a: ${ }^{1} \mathrm{H}$ NMR $\left(\mathrm{CDCl}_{3}\right): \delta 8.62(\mathrm{~s}, 1 \mathrm{H}), 8.56(\mathrm{~s}, 1 \mathrm{H})$, 7.78-7.68 (m, 2H), $7.38(\mathrm{t}, \mathrm{J}=7.6 \mathrm{~Hz}, 1 \mathrm{H}), 7.21(\mathrm{t}, \mathrm{J}=9.2 \mathrm{~Hz}, 1 \mathrm{H}), 4.51$ (br s, $1 \mathrm{H}), 3.36(\mathrm{t}, \mathrm{J}=7.2 \mathrm{~Hz}, 2 \mathrm{H}), 3.12-3.10(\mathrm{~m}, 2 \mathrm{H}), 1.82-1.75(\mathrm{~m}, 2 \mathrm{H})$, 1.58-1.37 (m, 6H), 1.43 (s, 9H); IR (KBr, $\left.\mathrm{cm}^{-1}\right): 3377,2934,1723,1692$, 1577, 1522, 1381, 1167, 899, 748, 633; Anal. Calcd. For $\mathrm{C}_{23} \mathrm{H}_{28} \mathrm{FN}_{5} \mathrm{O}_{3} \mathrm{~S}$ : C, 58.33; H, 5.96; F, 4.01; N, 14.79; O, 10.14; S, 6.77. Found: C, 57.87; H, 5.86; N, 14.59. (LC-MS) $\mathrm{m} / z: 474.11[\mathrm{M}+\mathrm{H}]^{+}$.

tert-butyl

6-(9-(2-methylbenzoyl)-9H-purin-6-ylthio) hexylcarbamate 8b: ${ }^{1} \mathrm{H}$ NMR $\left(\mathrm{CDCl}_{3}\right): \delta 8.73(\mathrm{~s}, 1 \mathrm{H}), 8.34(\mathrm{~s}, 1 \mathrm{H})$, 7.57-7.53 (m, 1H), 7.43-7.31 (m, 3H), $4.51(\mathrm{br} \mathrm{s}, 1 \mathrm{H}), 3.37(\mathrm{t}, \mathrm{J}=7.2 \mathrm{~Hz}$, $2 \mathrm{H}), 3.12-3.09(\mathrm{~m}, 2 \mathrm{H}), 2.46(\mathrm{~s}, 3 \mathrm{H}), 1.83-1.75(\mathrm{~m}, 2 \mathrm{H}), 1.61-1.33(\mathrm{~m}$, 6H), 1.43 (s, 9H); IR (KBr, cm-1): 3384, 2932, 1708, 1693, 1519, 1378, 1246, 1169, 897, 733, 633; Anal. Calcd. For $\mathrm{C}_{24} \mathrm{H}_{31} \mathrm{~N}_{5} \mathrm{O}_{3} \mathrm{~S}: \mathrm{C}, 61.38 ; \mathrm{H}$, $6.65 ; \mathrm{N}, 14.91$; O, 10.22; S, 6.83. Found: C, 60.29; H, 6.86; N, 14.89. (LCMS) $\mathrm{m} / z: 470.21[\mathrm{M}+\mathrm{H}]^{+}$.

tert-butyl 6-(9-(2-trifluoromethylbenzoyl)-9H-purin-6-ylthio) hexylcarbamate 8c: ${ }^{1} \mathrm{H}$ NMR $\left(\mathrm{CDCl}_{3}\right): \delta 8.55(\mathrm{~s}, 1 \mathrm{H}), 8.51(\mathrm{~s}, 1 \mathrm{H}), 7.86$ $(\mathrm{d}, \mathrm{J}=7.6 \mathrm{~Hz}, 1 \mathrm{H}), 7.79-7.70(\mathrm{~m}, 2 \mathrm{H}), 7.52(\mathrm{~d}, \mathrm{~J}=7.6 \mathrm{~Hz}, 1 \mathrm{H}), 4.49$ (br s, $1 \mathrm{H}), 3.35(\mathrm{t}, \mathrm{J}=7.2 \mathrm{~Hz}, 2 \mathrm{H}), 3.11-3.09(\mathrm{~m}, 2 \mathrm{H}), 1.81-1.73(\mathrm{~m}, 2 \mathrm{H}), 1.56-$ $1.36(\mathrm{~m}, 6 \mathrm{H}), 1.43(\mathrm{~s}, 9 \mathrm{H})$; IR $\left(\mathrm{KBr}, \mathrm{cm}^{-1}\right): 3392,2932,2860,1719,1569$, 1368, 1316, 1170, 1138, 900, 771, 634; Anal. Calcd. For $\mathrm{C}_{24} \mathrm{H}_{28} \mathrm{~F}_{3} \mathrm{~N}_{5} \mathrm{O}_{3} \mathrm{~S}$ : C, 55.06; H, 5.39; F, 10.89; N, 13.38; O, 9.17; S, 6.12. Found: C, 55.09; H, 5.26; N, 13.21. (LC-MS) $\mathrm{m} / z: 524.17[\mathrm{M}+\mathrm{H}]^{+}$.

tert-butyl 6-(9-(2-methoxylbenzoyl)-9H-purin-6-ylthio) hexylcarbamate 8d: ${ }^{1} \mathrm{H}$ NMR $\left(\mathrm{CDCl}_{3}\right): \delta 8.68(\mathrm{~s}, 1 \mathrm{H}), 8.39(\mathrm{~s}, 1 \mathrm{H})$, 7.65-7.61 (m, 2H), $7.15(\mathrm{t}, \mathrm{J}=7.6 \mathrm{~Hz}, 1 \mathrm{H}), 7.01(\mathrm{~d}, \mathrm{~J}=8.8 \mathrm{~Hz}, 1 \mathrm{H}), 4.50$ (br s, $1 \mathrm{H}), 3.62(\mathrm{~s}, 3 \mathrm{H}), 3.36(\mathrm{t}, \mathrm{J}=7.2 \mathrm{~Hz}, 2 \mathrm{H}), 3.12-3.10(\mathrm{~m}, 2 \mathrm{H}), 1.82-$ $1.79(\mathrm{~m}, 2 \mathrm{H}), 1.54-1.33(\mathrm{~m}, 6 \mathrm{H}), 1.43(\mathrm{~s}, 9 \mathrm{H})$; IR $\left(\mathrm{KBr}, \mathrm{cm}^{-1}\right)$ : 3380, 2936, 1742, 1689, 1581, 1520, 1387, 1180, 890, 752, 632; Anal. Calcd. For $\mathrm{C}_{24} \mathrm{H}_{31} \mathrm{~N}_{5} \mathrm{O}_{4} \mathrm{~S}$ : C, 59.36; H, 6.43; N, 14.42; O, 13.18; S, 6.60. Found: C, 59.21; H, 6.40; N, 14.31. (LC-MS) $\mathrm{m} / z: 486.26[\mathrm{M}+\mathrm{H}]^{+}$.

tert-butyl

6-(9-(4-methoxylbenzoyl)-9H-purin-6-ylthio) hexylcarbamate 8e: ${ }^{1} \mathrm{H}$ NMR $\left(\mathrm{CDCl}_{3}\right): \delta 8.73(\mathrm{~s}, 1 \mathrm{H}), 8.49(\mathrm{~s}, 1 \mathrm{H}), 7.88$ (d, J=8.8 Hz, 2H), $7.03(\mathrm{~d}, \mathrm{~J}=8.8 \mathrm{~Hz}, 2 \mathrm{H}), 4.52(\mathrm{br} \mathrm{s}, 1 \mathrm{H}), 3.93(\mathrm{~s}, 3 \mathrm{H})$, 3.39 (t, J=7.2 Hz, 2H), 3.13-3.11 (m, 2H), 1.84-1.77 (m, 2H), 1.54-1.38 (m, 6H), $1.44(\mathrm{~s}, 9 \mathrm{H})$; IR $\left(\mathrm{KBr}, \mathrm{cm}^{-1}\right): 3382,2928,1690,1557,1514$, 1368, 1264, 1172, 899, 762, 634; Anal. Calcd. For $\mathrm{C}_{24} \mathrm{H}_{31} \mathrm{~N}_{5} \mathrm{O}_{4} \mathrm{~S}$ : C, 59.36;
H, 6.43; N, 14.42; O, 13.18; S, 6.60. Found: C, 59.19; H, 6.42; N, 14.39. (LC-MS) $\mathrm{m} / z: 486.16[\mathrm{M}+\mathrm{H}]^{+}$.

tert-butyl 6-(9-(3-chlorobenzoyl)-9H-purin-6-ylthio) hexylcarbamate 8f: ${ }^{1} \mathrm{H}$ NMR $\left(\mathrm{CDCl}_{3}\right): \delta 8.70(\mathrm{~s}, 1 \mathrm{H}), 8.51(\mathrm{~s}, 1 \mathrm{H}), 7.85$ $(\mathrm{s}, 1 \mathrm{H}), 7.71(\mathrm{t}, \mathrm{J}=7.6 \mathrm{~Hz}, 1 \mathrm{H}), 7.53-7.49(\mathrm{~m}, 2 \mathrm{H}), 4.51(\mathrm{br} \mathrm{s}, 1 \mathrm{H}), 3.38(\mathrm{t}$, $\mathrm{J}=7.2 \mathrm{~Hz}, 2 \mathrm{H}), 3.12-3.10(\mathrm{~m}, 2 \mathrm{H}), 1.82-1.78(\mathrm{~m}, 2 \mathrm{H}), 1.55-1.37(\mathrm{~m}, 6 \mathrm{H})$, 1.43 (s, 9H); IR (KBr, cm $\left.{ }^{-1}\right): 3381,2942,2868,1714,1570,1342,1310$, 1178, 1136, 910, 774, 631; Anal. Calcd. For $\mathrm{C}_{23} \mathrm{H}_{28} \mathrm{ClN}_{5} \mathrm{O}_{3} \mathrm{~S}$ : C, 56.37; H, 5.76; Cl, 7.24; N, 14.29; O, 9.80; S, 6.54. Found: C, 56.30; H, 5.69; N, 14.18. (LC-MS) $\mathrm{m} / z: 490.12,492.14[\mathrm{M}+\mathrm{H}]^{+}$.

tert-butyl

6-(9-(1-naphthoyl)-9H-purin-6-ylthio) hexylcarbamate 8g: ${ }^{1} \mathrm{H}$ NMR $\left(\mathrm{CDCl}_{3}\right): \delta 8.70(\mathrm{~s}, 1 \mathrm{H}), 8.55(\mathrm{~s}, 1 \mathrm{H}), 8.40$ $(\mathrm{s}, 1 \mathrm{H}), 8.02-7.91(\mathrm{~m}, 4 \mathrm{H}), 7.72-7.62(\mathrm{~m}, 2 \mathrm{H}), 4.51(\mathrm{br} \mathrm{s}, 1 \mathrm{H}), 3.40(\mathrm{t}$, $\mathrm{J}=7.2 \mathrm{~Hz}, 2 \mathrm{H}), 3.13-3.11(\mathrm{~m}, 2 \mathrm{H}), 1.84-1.80(\mathrm{~m}, 2 \mathrm{H}), 1.56-1.38(\mathrm{~m}, 6 \mathrm{H})$, 1.44 (s, 9H); IR $\left(\mathrm{KBr}, \mathrm{cm}^{-1}\right): 3293,2929,1689,1558,1369,1283,1190$, 911, 806, 756, 633; Anal. Calcd. For $\mathrm{C}_{27} \mathrm{H}_{31} \mathrm{~N}_{5} \mathrm{O}_{3} \mathrm{~S}: \mathrm{C}, 64.14 ; \mathrm{H}, 6.18 ; \mathrm{N}$, 13.85; O, 9.49; S, 6.34. Found: C, 64.02; H, 6.21; N, 13.59. (LC-MS) m/z: $506.16[\mathrm{M}+\mathrm{H}]^{+}$.

tert-butyl 6-(9-propionoyl-9H-purin-6-ylthio)hexylcarbamate 8h: ${ }^{1} \mathrm{H}$ NMR $\left(\mathrm{CDCl}_{3}\right): \delta 8.77(\mathrm{~s}, 1 \mathrm{H}), 8.69(\mathrm{~s}, 1 \mathrm{H}), 4.51$ (br s, $\left.1 \mathrm{H}\right), 3.47$ (q, J=7.2 Hz, 2H), 3.37 (t, J=7.6 Hz, 2H), 3.12-3.10 (m, 2H), 1.83-1.79 (m, 2H), 1.56-1.34 (m, 9H), 1.44 (s, 9H); IR $\left(\mathrm{KBr}, \mathrm{cm}^{-1}\right)$ : 3371, 2921, 1748, 1675, 1563, 1516, 1366, 1240, 1183, 909, 729, 602; Anal. Calcd. For $\mathrm{C}_{19} \mathrm{H}_{29} \mathrm{~N}_{5} \mathrm{O}_{3} \mathrm{~S}$ : C, 56.00; H, 7.17; N, 17.18; O, 11.78; S, 7.87. Found: C, 56.09; H, 7.11; N, 17.02. (LC-MS) $\mathrm{m} / z: 408.09[\mathrm{M}+\mathrm{H}]^{+}$.

tert-butyl 6-(9-(3-methylbutanoyl)-9H-purin-6-ylthio) hexylcarbamate 8i: ${ }^{1} \mathrm{H}$ NMR $\left(\mathrm{CDCl}_{3}\right): \delta 8.77(\mathrm{~s}, 1 \mathrm{H}), 8.67(\mathrm{~s}, 1 \mathrm{H}), 4.51$ (br s, $1 \mathrm{H}), 3.39-3.33(\mathrm{~m}, 4 \mathrm{H}), 3.12-3.10(\mathrm{~m}, 2 \mathrm{H}), 2.35-2.32(\mathrm{~m}, 1 \mathrm{H})$, 1.81-1.75 (m, 2H), 1.54-1.37 (m, 6H), $1.44(\mathrm{~s}, 9 \mathrm{H}), 1.09(\mathrm{~d}, \mathrm{~J}=6.8 \mathrm{~Hz}$, $6 \mathrm{H})$; IR $\left(\mathrm{KBr}, \mathrm{cm}^{-1}\right): 3379,2934,1732,1689,1527,1198,1170,916,639$; Anal. Calcd. For $\mathrm{C}_{21} \mathrm{H}_{33} \mathrm{~N}_{5} \mathrm{O}$ S: C, 57.91; H, 7.64; N, 16.08; O, 11.02; S, 7.36. Found: C, 57.89; H, 7.56; N, 16.11. (LC-MS) m/z: $436.25[\mathrm{M}+\mathrm{H}]^{+}$.

Synthesis of 6-(9H-purin-6-ylthio) hexan-1-amine 9: To a stirred solution of compound $8 \mathrm{a}(200 \mathrm{mg}, 0.42 \mathrm{mmol})$ in Dichloromethane (3 $\mathrm{ml})$ was added TFA $(0.034 \mathrm{~mL}, 0.42 \mathrm{mmol})$ at $0^{\circ} \mathrm{C}$ for $30 \mathrm{~min}$. Reaction was monitored by TLC. When reaction was completed, the reaction mixture was concentrated under reduced pressure. Crude compound was purified by column chromatography to get compound 9 as brown colour solid (70 mg, 66\%). The same result was obtained from Dioxane$\mathrm{HCl}$ method, and spectral data indicates absence of compound $9 \mathrm{a} .{ }^{1} \mathrm{H}$ NMR (DMSO-d ): $\delta 8.68(\mathrm{~s}, 1 \mathrm{H}), 8.44(\mathrm{~s}, 1 \mathrm{H}), 4.2(\mathrm{br} \mathrm{s}, 2 \mathrm{H}), 3.35(\mathrm{t}$, $\mathrm{J}=6.8 \mathrm{~Hz}, 2 \mathrm{H}), 2.79-2.75(\mathrm{~m}, 2 \mathrm{H}), 1.75-1.68(\mathrm{~m}, 2 \mathrm{H}), 1.59-1.36(\mathrm{~m}, 6 \mathrm{H})$ (LC-MS) $\mathrm{m} / z: 252.21[\mathrm{M}+\mathrm{H}]^{+}$.

\section{Results and Discussions}

The synthesis of 9-substituted derivatives of tert-butyl 6-(9H-purin6-ylthio) hexylcarbamate ( $8 \mathrm{a}-8 \mathrm{i})$ is described in scheme 1. Compound 3 was prepared by the Boc protection of 6 -amino- hexanol and then followed by mesylation [11] in THF as a solvent (Scheme 2). 6-chloro purine was reacted with potassium thioacetate in DMF at $80^{\circ} \mathrm{C}$ for 6 $\mathrm{h}$ to get 6-mercaptopurine [12]. The obtained 6-mercaptopurine was reacted with compound 3; the reaction was carried out in anhydrous DMF in the presence of one equivalent of anhydrous potassium carbonate at room temperature for $16 \mathrm{~h}$ to get mixture of only mono alkylated as major, di-alkylated derivative as minor amounts 6 and 7 . Another one more reaction was tried in two equivalent of anhydrous potassium carbonate to get 1: 1 ratio of mono and di-alkylated compounds. Compound 6 was reacted with different acid chlorides in presence of Triethylamine and DCM to afford the titled compounds 

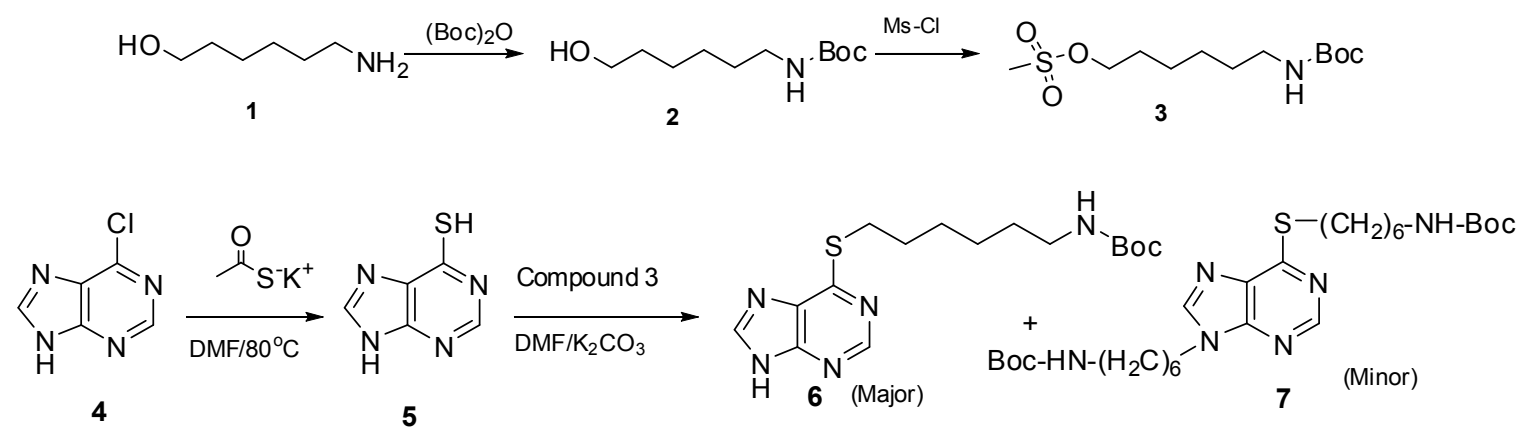<smiles>[R]C(=O)OCCOCCO</smiles><smiles>[R]C(=O)n1cnc2c(SCCCCCCNC(C)=O)ncnc21</smiles>

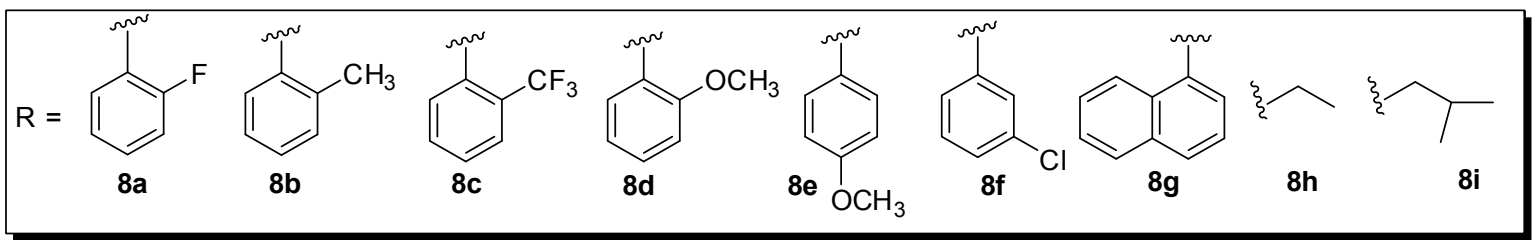

Scheme 1: Synthesis of 9-substituted tert-butyl 6-(9H-purin-6-ylthio) hexylcarbamate derivatives.<smiles>CC(C)C(=O)NCCCCCCSc1ncnc2c1ncn2C(=O)c1ccccc1F</smiles><smiles>NCCCCCCSc1ncnc2[nH]cnc12</smiles>

Scheme 2: Boc deprotection of 9-substituted tert-butyl 6-(9H-purin-6-ylthio) hexylcarbamate derivative.

\begin{tabular}{|c|c|c|c|c|}
\hline compound & Molecular formula & Molecular weight & Melting range ${ }^{\circ} \mathrm{C}$ & Yield (\%) \\
\hline $8 a$ & $\mathrm{C}_{23} \mathrm{H}_{28} \mathrm{FN}_{5} \mathrm{O}_{3} \mathrm{~S}$ & 473.56 & $79-81$ & 56 \\
\hline $8 b$ & $\mathrm{C}_{24} \mathrm{H}_{31} \mathrm{~N}_{5} \mathrm{O}_{3} \mathrm{~S}$ & 469.60 & $98-101$ & 58 \\
\hline $8 c$ & $\mathrm{C}_{24} \mathrm{H}_{28} \mathrm{~F}_{3} \mathrm{~N}_{5} \mathrm{O}_{3} \mathrm{~S}$ & 523.57 & $52-54$ & 54 \\
\hline $8 d$ & $\mathrm{C}_{24} \mathrm{H}_{31} \mathrm{~N}_{5} \mathrm{O}_{4} \mathrm{~S}$ & 485.60 & $84-86$ & 62 \\
\hline $8 e$ & $\mathrm{C}_{24} \mathrm{H}_{31} \mathrm{~N}_{5} \mathrm{O}_{4} \mathrm{~S}$ & 485.60 & $78-80$ & 64 \\
\hline $8 f$ & $\mathrm{C}_{23} \mathrm{H}_{28} \mathrm{CIN}_{5} \mathrm{O}_{3} \mathrm{~S}$ & 490.02 & $100-102$ & 53 \\
\hline $8 g$ & $\mathrm{C}_{27} \mathrm{H}_{31} \mathrm{~N}_{5} \mathrm{O}_{3} \mathrm{~S}$ & 505.63 & $115-118$ & 61 \\
\hline $8 \mathrm{~h}$ & $\mathrm{C}_{19} \mathrm{H}_{29} \mathrm{~N}_{5} \mathrm{O}_{3} \mathrm{~S}$ & 407.53 & $97-99$ & 51 \\
\hline $8 \mathrm{i}$ & $\mathrm{C}_{21} \mathrm{H}_{33} \mathrm{~N}_{5} \mathrm{O}_{3} \mathrm{~S}$ & 435.58 & $92-94$ & 62 \\
\hline
\end{tabular}

Table 1: Physical data of the newly synthesized 9-substituted derivatives of tert-butyl 6-(9H-purin-6-ylthio) hexylcarbamate (8a-8i).

of 9-substituted derivatives of tert-butyl 6-(9H-purin-6-ylthio) hexylcarbamate $(8 \mathrm{a}-8 \mathrm{i})$ in good yields. The purity of compounds $8 \mathrm{a}-8 \mathrm{i}$ was confirmed by using TLC, LCMS purity and their NMR spectral data. In other side we are tried for the deprotection of Boc group of compound 8a by using different conditions like Trifluoroacetic acid in Dichloromethane, Dioxane- $\mathrm{HCl}$ at cold condition causes deprotection not only of the Boc group but also fission of acylated derivative to give the 6-(9H-purin-6-ylthio)hexan-1-amine 9. The compound 9 was 
Citation: Venkateswara Rao P, Ravindhranath K, Ravi Kumar K (2013) Synthesis of 9-Substituted Derivatives of tert-Butyl 6-(9h-Purin-6-Ylthio) Hexylcarbamate. Med chem 3: 188-191. doi:10.4172/2161-0444.1000137

confirmed by ${ }^{1} \mathrm{H}$ NMR and Mass spectral data. The obtained result indicates that the derivatives of 9-substituted tert-butyl 6-(9H-purin6-ylthio) hexylcarbamate are acid sensitive (Table 1).

\section{Conclusion}

In conclusion, we have synthesized 9-substituted derivatives of tert-butyl 6-(9H-purin-6-ylthio) hexylcarbamate in good yields and characterized by ${ }^{1} \mathrm{H}$ NMR, IR and LCMS spectral data.

\section{Acknowledgments}

The authors are very much grateful to the management of R.V.R \& J.C College of Engineering, Guntur, A.P, India, for providing moral support in carrying out this work.

\section{References}

1. Rida SM, Ashour FA, El-Hawash SA, El-Semary MM, Badr MH (2007) Synthesis of some novel substituted purine derivatives as potential anticancer anti-HIV-1 and antimicrobial agents. Arch Pharm (Weinheim) 340: 185-194.

2. Lech-Maranda E, Korycka A, Robak T (2006) Pharmacological and clinical studies on purine nucleoside analogs--new anticancer agents. Mini Rev Med Chem 6: 575-581.

3. Steurer M, Pall G, Richards S, Schwarzer G, Bohlius J, et al. (2006) Single- agent purine analogues for the treatment of chronic lymphocytic leukaemia: a systematic review and meta-analysis. Cancer Treat Rev 32: 377-389.

4. Zaza G, Yang W, Kager L, Cheok M, Downing J, et al. (2004) Acute lymphoblastic leukemia with TEL-AML1 fusion has lower expression of genes involved in purine metabolism and lower de novo purine synthesis. Blood 104: 1435-1441.

5. Robak T, Lech-Maranda E, Korycka A, Robak E (2006) Purine nucleoside analogs as immunosuppressive and antineoplastic agents: mechanism of action and clinical activity. Curr Med Chem 13: 3165-3189.

6. Schwartz RS (2000) Immunosuppression-back to the future. World J Surg 24 783-786.

7. Carey NH, Mandel HG (1961) Studies on the inhibition of growth of Bacillus cereus by 6-mercaptopurine. J Biol Chem 236: 520-524.

8. Mars U, Larsson BS (1995) New thioureas and related substances intended for melanoma targeting. Pigment Cell Res 8: 194-201.

9. Rubina K, Abele E, Arsenyan P, Abele R, Veveris M, et al. (2001) Synthesis of silicon and germanium containing heteroaromatic sulfides as cholesterol level lowering and vasodilating agents. Metal Based Drugs 8: 85-93.

10. Neiman Z, Bergmann P (1965) Israel J Chem 3:161.

11. Bergbreiter DE, Osburn PL, Li C (2002) Soluble polymer-supported catalysts containing azo dyes. Org Lett 4: 737-740.

12. Sariri R, Khalili G, Russian (2002) J Org Chem 38: 1053. 